\title{
Stable Aqueous Suspension and Self-Assembly of Graphite Nanoplatelets Coated with Various Polyelectrolytes
}

\author{
Jue Lu, ${ }^{1,2}$ Inhwan Do, ${ }^{1}$ Hiroyuki Fukushima, ${ }^{1}$ Ilsoon Lee, ${ }^{2}$ and Lawrence T. Drzal ${ }^{1}$ \\ ${ }^{1}$ Composite Materials and Structures Center, Michigan State University, East Lansing, MI 48824-1226, USA \\ ${ }^{2}$ Department of Chemical Engineering and Materials Science, Michigan State University, East Lansing, MI 48824-1226, USA
}

Correspondence should be addressed to Ilsoon Lee, leeil@msu.edu and Lawrence T. Drzal, drzal@egr.msu.edu

Received 1 March 2010; Accepted 7 May 2010

Academic Editor: Rakesh Joshi

Copyright ( $) 2010$ Jue Lu et al. This is an open access article distributed under the Creative Commons Attribution License, which permits unrestricted use, distribution, and reproduction in any medium, provided the original work is properly cited.

\begin{abstract}
Exfoliated graphite nanoplatelets (xGnPs) with an average thickness of $1-10 \mathrm{~nm}$ present an inexpensive alternative to carbon nanotubes in many applications. In this paper, stable aqueous suspension of $\mathrm{xGnP}$ was achieved by noncovalent functionalization of $\mathrm{xGnP}$ with polyelectrolytes. The surfactants and polyelectrolytes were compared with respect to their ability to suspend graphite nanoplatelets. The surface charge of the nanoplatelets was characterized with zeta potential measurements, and the bonding strength of the polymer chains to the surface of $\mathrm{xGnP}$ was characterized with Raman spectroscopy. This robust method opens up the possibility of using this inexpensive nanomaterial in many applications, including electrochemical devices, and leads to simple processing techniques such as layer-by-layer deposition. Therefore, the formation of xGnP conductive coatings using layerby-layer deposition was also demonstrated.
\end{abstract}

\section{Introduction}

Considerable interest has been drawn in the layer-by-layer (LbL) self-assembly of nanosized carbon particles due to their excellent thermal, electrical, and mechanical properties [1]. Carbon nanotubes (CNTs) and fullerenes (also known as buckyball or $\mathrm{C}_{60}$ ) have been intensively studied, and their possible applications in nanodevices $[2,3]$, quantum wires [4], ultrahigh-strength engineering fibers [5], sensors $[6,7]$, and support of electrocatalysts [4] are developing. Both carbon nanotubes and fullerenes are expensive, which hinders their application in industry. Recently, the twodimensional carbon graphene has attracted great attention, due to its exceptionally high crystal and electronic quality $[8,9]$. Growth of large area of high-quality graphene was developed on metal substrates [10], and individual graphene sheets can be also prepared by micromechanical cleavage [8]. Unfortunately, these approaches are technically complex and difficult for mass production. In addition, these twodimensional crystals cannot theoretically exist in the free state [8]. The most commonly used nano sized form of graphene is graphite nanoplatelets, which are a few layers of graphene sheets stacked together and are produced by the exfoliation of graphite via an acid intercalation, followed by ultrasonic irradiation to isolated graphite nanosheets [11]. Research in our group at MSU has led to a process that can successfully produce exfoliated graphite nanoplatelets $\left(\mathrm{xGnP}^{\mathrm{TM}}\right)$, which are $1-10 \mathrm{~nm}$ in thickness and from 100 to $1000 \mathrm{~nm}$ in diameter. The surface of the graphite nanoplatelets is pure graphene where the carbon is in a sp ${ }^{2}$ configuration. This presents a uniformly homogeneous and moderately energetic surface that has been shown to be an excellent nucleating surface for both polar and nonpolar polymers. Furthermore, these graphite nanoplatelets possess the high electric conductivity of graphene. With an expected cost on the order of $\$ 5 /$ pound, these nanoplatelets could be a suitable substitute for carbon nanotubes and fullerenes for most applications. So far, graphite nanoplatelets are most commonly used in composite application due to their good mechanical properties and electrical conductivity [1214]. Recently, we have demonstrated that $\mathrm{xGnP}$ can be an inexpensive alternative to carbon nanotubes and carbon black as an advantageous support for fuel cell applications, which has the highest thermal-oxidation resistance and the highest degree of graphitization [15]. In addition, $\mathrm{xGnP}$ and $\mathrm{xGnP}$-supported electrocatalysts have shown significant catalytic effect toward hydrogen peroxide, resulting highly sensitive and quickly responding glucose biosensors $[16,17]$. 
The major challenge for these hydrophobic carbon nanostructures is their dispersion in an aqueous medium. Several approaches have been studied for the production of aqueous suspension of carbon nanotubes. Chemical modification of the graphene surface is common, such as acid treatment, which imparts - $\mathrm{COOH}$ groups at the broken links or at the ends of nanotubes $[18,19]$. However, such chemical modification can disrupt the electronic conjugation in carbon nanotubes which inevitably deteriorate the electrical properties of the nanotubes. Another common method is to suspend carbon nanotubes in various surfactants or charged polymers accompanied by the physical processes, which involves high-shear homogenization and ultrasonication [20]. This method became universal for carbon nanotube dispersion because it is easy to control, and most importantly, it preserves the integrity of carbon nanotubes. The functionalized carbon nanotubes are used to construct thin films for various applications.

Since Decher's pioneering work in 1997 [21], the LbL assembly method has been well studied to create highly tuned, functional thin films with nanometer-level thickness [1]. In LbL assembly, oppositely charged polymers are alternately adsorbed on the surface by the electrostatic force, although possible hydrogen bonding, hydrophobic interaction, and van der Waals interactions could also be used. Considerable work has been done on self-assembly of carbon nanotubes. This approach can solve the dispersion problems of carbon nanotubes in polymer matrix, one of the big challenges of the traditional composite processing method. In addition, LbL assembly was also used to incorporate carbon nanotubes in the construction of chemical sensors and biosensors, due to their high electrocatalytic effect, fast electron transfer, rate and large working surface area [22]. Several groups have reported the preparation of selfassembled carbon films using graphite oxide nanoplatelets [23-26]. However, the oxidation of graphite reduced the original conductivity of graphite. To restore the conductivity of the carbon films, the graphite oxide needs to be reduced to the original state by either chemical or electrochemical methods, which is not cost effective. In addition, this additional process may inhibit their use in biosensor application due to the enzyme stability during the reduction process.

In this paper, we report the achievement of stable aqueous dispersions of graphite nanoplatelets and their selfassembly. Since graphite nanoplatelets are not stable in aqueous solution due to the hydrophobic characteristics of graphene sheets and their significant hydrophobic interactions which form agglomerates in aqueous solution. Much effort has been devoted to achieve a stable suspension of graphite nanoplatelets with various surfactants and polyelectrolytes (PEs). In addition, by modifying the charge of polyelectrolytes on the graphite sheet, the agglomeration of graphite nanoplatelets can be possibly prevented by electrostatic or strong repulsive forces due to the surface charge induced by the polyelectrolytes during the self-assembly process. The morphologies of the graphite monolayer and multilayer films have been characterized by the optical microscopy $(\mathrm{OM})$, scanning electron microscopy (SEM) and atomic force microscopy (AFM). Additionally, the resistance and transmittance of these films were also measured.

\section{Experimental Procedures}

2.1. Materials. Poly(diallyldimethylammonium chloride) (PDAC), sulfated poly(styrene) (SPS), and branched poly ethyleimine (PEI) were purchased from Aldrich. The weight average molecular weights $\left(M_{w}\right)$ of PDAC, SPS, and PEI were $\sim 100,000-200,000,70,000$, and 750,000, respectively. Polyacrylic acid (PAA) $\left(M_{w}=90,000\right)$ was purchased from Polysciences Inc. Poly(3,4-ethylenedioxythiophene) poly(styrene sulfonate) (PEDT/SPS) (Baytron P V407) was received as a sample from Bayer Corp. and used as received. The surfactants, sodium dodecylbenzene sulfonate (SDBS) and sodium dodecyl sulfate (SDS) were purchased from Aldrich and used as received. Microscope glass slides were purchased from Corning. All aqueous solutions in the processes were prepared using deionized (DI) water $(>18.1 \mathrm{M} \Omega$ ) supplied by a Barnstead Nanopur Diamond-UV purification unit equipped with a UV source and final $0.2 \mu \mathrm{m}$ filter. All procedures were done at room temperature.

\subsection{Preparation of Polyelectrolyte and Surfactant Solutions.} Aqueous polyelectrolyte solutions were prepared containing either $20 \mathrm{mM}$ PDAC or $10 \mathrm{mM}$ SPS in $0.1 \mathrm{M} \mathrm{NaCl}$. PEI and PAA solution was prepared containing $0.1 \mathrm{wt} \%$ polymer in DI water, and the $\mathrm{pH}$ value of the solution was not adjusted. The solutions were filtered with $0.22 \mu \mathrm{m}$ cellulose acetate membrane filter system from Corning to remove particulates. Other solutions with surfactants (SDBS and SDS) were prepared containing $0.1 \mathrm{wt} \%$ surfactants in DI water.

2.3. Preparation of Graphite Suspension. $0.1 \mathrm{~g}$ of $\mathrm{xGnP}$ was dispersed in $100 \mathrm{~mL}$ polyelectrolyte solution by sonication with an output power of $23 \mathrm{~W}$ for 30 minutes. The suspension was stirred for 24 hours. The excess polyelectrolyte was removed by filtering through $0.2 \mu \mathrm{m}$ membrane followed by DI water washing for three times. The polyelectrolyte-coated $\mathrm{xGnP}$ was redispersed to $100 \mathrm{~mL}$ DI water by mild sonication (output power: $10 \mathrm{~W}$ ) for $10-15$ minutes. The $\mathrm{pH}$ value of the suspension with PEI-coated $\mathrm{xGnP}$ was adjusted to be between 5.0-6.0.

2.4. Layer-by-Layer Self-Assembly Procedure. To prepare $\mathrm{xGnP} /$ polyelectrolyte multilayers, anionic PEs-xGnP such as SPS-xGnP were alternately assembled with PDAC, and cationic PEs-xGnP, such as PEI-xGnP with SPS. First, the glass slides were cleaned twice in an ultrasonic unit, first with a commercially available detergent (Alconox, Alconox Inc.) for 20 minutes and then without for 10 minutes. Slides were dried under a $\mathrm{N}_{2}$ gas stream and then treated with oxygen plasma for 10 minutes at 150 mTorr vacuum to activate negative surface charges on the glass. The multilayers of $\mathrm{xGnP}$ were prepared using a Microm DS 50 Slide Stainer purchased from Richard-Allan Scientific. The pretreated glass slides were immersed in the PDAC solution 
for 20 minutes, followed by two 5-minutes rinses in DI water. Then the glass slides were immersed in graphite suspension for $30 \mathrm{~min}$, followed by three 2-minutes rinses in DI water. The immersion time in $\mathrm{xGnP}$ is considered sufficient since longer time did not increase the surface coverage of $\mathrm{xGnP}$. The sequence was repeated until the desired number of bilayers was formed. The substrates were dried naturally.

\subsection{Characterization of $x G n P$ and Polymer-Modified $x G n P$.} Transmission electron microscopy (TEM) investigation was carried out with JEOL 100CX and JEOL 2200FS operating at a voltage of $100 \mathrm{keV}$ and $200 \mathrm{keV}$ for the morphology of $\mathrm{xGnP}$. Specimens were prepared by ultrasonically dispersing the sample powders in acetone for 15 minutes, applying the powder suspension onto lacey carbon-coated $\mathrm{Cu}$ grids and drying them in air at ambient temperature. An X-ray photoelectron spectroscopy spectrum (XPS, Physical Electronics 5400 ESCA) was used to study the surface of $\mathrm{xGnP}$ and polymer modified $\mathrm{xGnP}$. UV-vis absorption measurement was taken on a Perkin Elmer UV/Vis/NIR spectrometer (model Lamda 900). Thermogravimetric analysis (TGA) was performed using a TGA 2950 (TA instruments) from $25^{\circ} \mathrm{C}$ to $580^{\circ} \mathrm{C}$ at a heating rate of $10.0^{\circ} \mathrm{C} / \mathrm{min}$ under an air flow. Approximately $10 \mathrm{mg}$ of $\mathrm{xGnP}$ was used.

For Raman measurements, the $\mathrm{xGnP}$ thin films were prepared by filtering a certain amount of $\mathrm{xGnP}$ and polymercoated $\mathrm{xGnP}$ on cellulose ester membrane with a pore size of $0.2 \mu \mathrm{m}$, and drying for a few days. The films were peeled off from the membrane before Raman measurement. The Raman characterization was carried out on a micro-Raman system with a laser wavelength of $532 \mathrm{~nm}$ and an intensity of $14.5 \mathrm{~mW}$.

Both Zeta potential and particle size of $\mathrm{xGnP}$ were analyzed with Brookhaven Instruments ZetaPALS, which utilizes phase analysis light scattering (PALS) to determine the electrophoretic mobility of charged colloidal suspensions. The velocities of the charged particles were measured and the electrophoretic mobility was determined by dividing the measured velocity by the electric field strength. The zeta potential was determined from the electrophoretic mobility using the Smoluchowski equation. Before measurement, the polymer coated graphite nanoplatelets were suspended in DI water by sonication. The particle size was also analyzed with ZetaPALS incorporated with a 90Plus particle sizer. Since the principles of dynamic light scattering assume the particles to be spherical, only relative values of particle size were obtained. Each value of particle size is the average of ten measurements of the sample. For comparison, AcoustoSizer IIs (Colloidal Dynamics) was also used for measuring the particle size of $x G n P .120 \mathrm{~mL}$ of $\mathrm{xGnP}$ suspension with $1 \mathrm{wt} \%$ solid content was used.

2.6. Multilayers Characterizations. The morphology of single and multilayer films of $\mathrm{xGnP}$ on glass slides was characterized using scanning electron microscopy (SEM, JEOL 6300F). A layer of osmium was coated with a pure osmium coater (NEOC-AN, MEIWA SHOJI CO. LTD, JAPAN) for $20 \mathrm{~s}$ for enhanced conductivity before SEM measurements.
The morphology of multilayer was also characterized using atomic force microscopy (AFM, a Nanoscope IV version from Veeco Instruments (Santa Barbara, CA)) in tapping mode. The surface resistance of $\mathrm{xGnP}$ multilayer was measured with a Camry Instruments Femtostat Station, and the transmittance was measured with Perkin Elmer Lambda 900 UV/Vis/NIR spectrometer.

\section{Results and Discussions}

Graphite has a layered structure with hybridized carbon atoms in an $\mathrm{sp}^{2}$ configuration, and different layers are held together by van der Waals forces. Exfoliation of graphite is achieved by intercalation compounds which tend to exfoliate graphite upon heating due to the fast evaporation of intercalantes. A process was developed in our lab to produce exfoliated graphite nanoplatelets from a sulfuric acid-based intercalated graphite by microwave and sonication process, followed by a milling process to further reduce the particle size to approximately $1 \mu \mathrm{m}$. BET surface area analysis showed an area of approximately $100 \mathrm{~m}^{2} / \mathrm{g}$ for the produced exfoliated graphite nanoplatelets [27]. Since a hypothetical monolayer graphite would exhibit a specific surface area close to $2700 \mathrm{~m}^{2} / \mathrm{g}$, and an interlayer spacing of $0.335 \mathrm{~nm}$ is assumed [28], the average thickness of graphite nanoplatelets was estimated to be $1-10 \mathrm{~nm}$, which was further confirmed by TEM observation. As shown in Figure 1, the top view of $x G n P$ shows clean surface of $x G n P$. It seems graphene sheets with different size are stacking together. Side view of $\mathrm{xGnP}$ clearly shows about 30 layers of atomic sheets stacking together, which counts to the thickness up to $10 \mathrm{~nm}$.

To eliminate the need to search wide availability of surfactants and polyelectrolytes, the literature results of their ability for solublizing carbon nanotubes were adapted in this work. Therefore, a series of good surfactants and polyelectrolytes for carbon nanotubes were chosen to test for their ability to suspend and then self-assemble $\mathrm{xGnP}$ on the charged glass slides. They include sodium dodecylbenzene sulfonate (SDBS), sodium dodecyl sulfate (SDS), sulfated poly(styrene) (SPS), poly(acrylic acid) (PAA), and a conductive polymer blend: poly(3,4-ethylenedioxythiophene) poly(styrene sulfonate) (PEDT/SPS) was also used. In addition, a couple of positive polyelectrolytes, poly(diallyldimethylammonium chloride) (PDAC) and branched polyethyleimine (PEI) were also tested. Table 1 shows the summary of quick trial-and-error experiments. $\mathrm{xGnP}$ was dispersed in solution with the surfactants or polyelectrolytes and their stability was checked after 24 hours. The suspension with SDBS and SDS was relatively stable but self-assembly of $x \mathrm{GnP}$ resulted poor surface coverage of particles. Therefore, further study was not conducted on these surfactants. PDAC and PAA also showed poor dispersing ability. It was found that SPS and PEDT/SPS coated $\mathrm{xGnP}$ showed good stability in water. This is possibly due to the edge-to-face interactions between the graphitic surface and the aromatic rings of the polymer [29]. PEI is also a good suspending agent due to the hydrophobic interaction between PEI and uncharged graphitic surface [30]. 
TABLE 1: General performance of surfactants and polyelectrolytes on the solublization of xGnP and layer-by-layer deposition thereafter.

\begin{tabular}{lccc}
\hline & & Suspension After 24 hours & Layer-by-layer deposition \\
\hline \multirow{2}{*}{ Surfactants } & SDS & Partially settle down & Poor \\
& SDBS & Partially settle down & Poor \\
\hline \multirow{3}{*}{ Polyelectrolytes } & PDAC & Mostly settle down & Poor \\
& PEI & Stable & Good \\
& SPS & Stable & Good \\
& PEDT/SPS & Stable & Poor \\
\hline
\end{tabular}

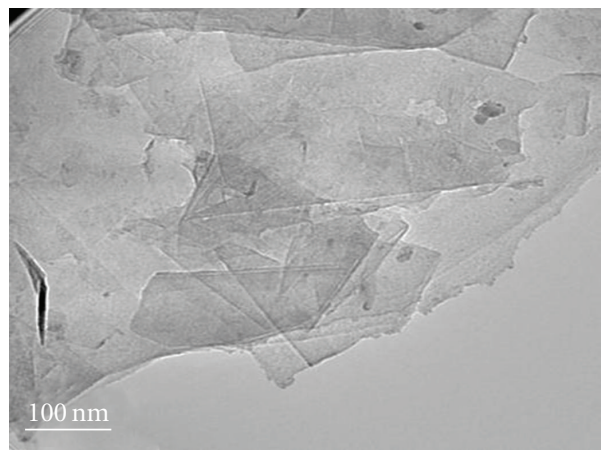

(a)

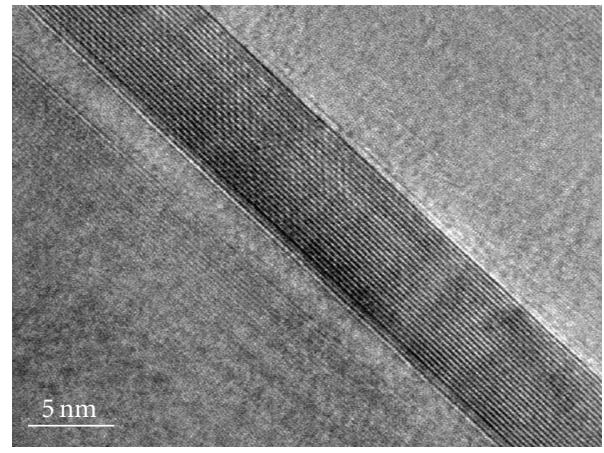

(b)

FIgUre 1: Typical TEM images of xGnP: (a) top view and (b) side view.

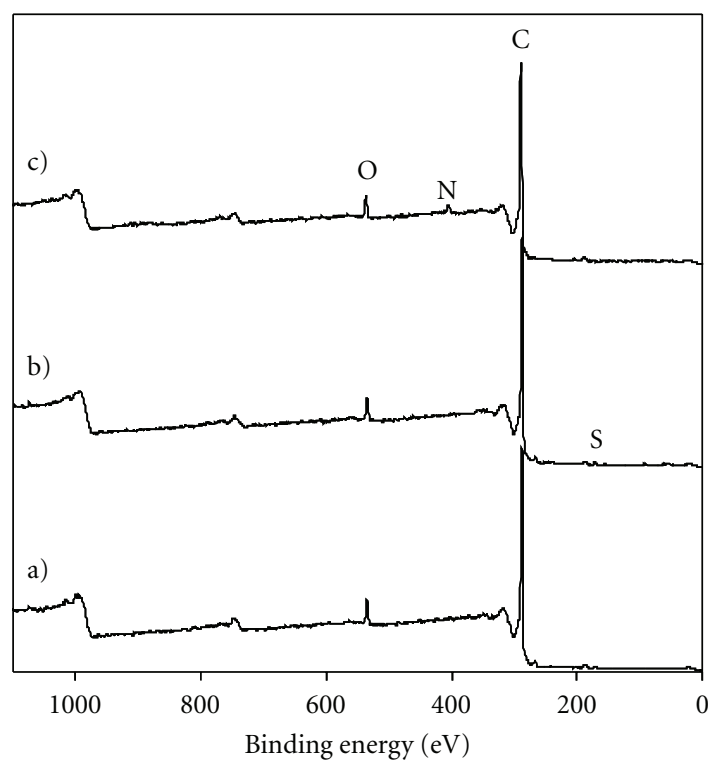

FIGURE 2: X-ray photoelectron spectroscopy spectra of $\mathrm{xGnP}$ before (a) and after SPS (b) and PEI (c) modification.

XPS was used to study the surface of $\mathrm{xGnP}$ before and after polymer coating. As shown in Figure 2, the surface of $\mathrm{xGnP}$ mainly contains $\mathrm{C} 1 \mathrm{~s}$ which shows peaks in the range of 280-290 eV. Small amount of oxygen is also shown which has an $\mathrm{O} 1 \mathrm{~s}$ peak at $540 \mathrm{eV}$. The oxygens mostly come from the edge group of the $\mathrm{xGnP}$ sheets such as carboxyl, hydroxyl, and carbonyl groups. There is also some $\mathrm{Zr}$ shown mainly due to the contamination of zirconium ball during the milling process. The SPS coated $\mathrm{xGnP}$ shows its characteristic peaks of S2p at $167.3 \mathrm{eV}$, corresponding to sulfone. PEI coated $\mathrm{xGnP}$ shows N1s peak at $401 \mathrm{eV}$. Therefore, XPS clearly shows the bonding of the polyelectrolyte to the graphite surface. Table 2 shows the detailed concentration of atoms for $\mathrm{xGnP}$ with and without polymer coatings. The amount of polymer coated on the surface is low. TGA was used to directly measure the amount of polymer coated on the $\mathrm{xGnP}$. As shown in Figure 3, graphite is thermally stable in ambient air environments up to $500^{\circ} \mathrm{C}$, and then it slightly decomposed at elevated temperature. The SPS coated $\mathrm{xGnP}$ shows a gradual loss due to the residue water, and a major degradation starts at approximately $360^{\circ} \mathrm{C}$. Instead, both PEI and PDAC coated $\mathrm{xGnP}$ show the major degradation approximately at $200^{\circ} \mathrm{C}$, which are much less thermally stable than SPS coated $\mathrm{xGnP}$. It is clear that SPS has higher amount of coating on $\mathrm{xGnP}$ than PEI and PDAC, which is approximately $5 \mathrm{wt} \%$. Stankovich et al. claimed that $\sim 40 \mathrm{wt} \%$ of SPS is coated on graphite nanoplatelets when dispersing nanoplatelets with a thickness of $\sim 4 \mathrm{~nm}$ [29]. Various concentrations of SPS and coating times were used to increase the coverage of polymer on $\mathrm{xGnP}$, but no increase in SPS content on $\mathrm{xGnP}$ was observed.

The surface charges of polyelectrolytes coated $\mathrm{xGnP}$ and unmodified $\mathrm{xGnP}$ were characterized using a zeta potential analyzer. The zeta potential describes the nature of the electrostatic potential near the surface of a suspended particle. In general, agglomeration of particles could be avoided by electrostatic repulsion above certain surface potentials, which is approximately $\pm 35 \mathrm{mV}$; and, the higher the absolute 
TABLE 2: Atomic concentration of the surface of xGnP before and after PE modification.

\begin{tabular}{|c|c|c|c|c|c|}
\hline & $\mathrm{C} 1 \mathrm{~s}$ & O1s & S2p & N1s & $\mathrm{Zr3d}$ \\
\hline $\mathrm{xGnP}$ & 92.38 & 6.38 & & & 0.25 \\
\hline SPS-xGnP & 94.31 & 4.51 & 0.76 & & 0.22 \\
\hline SPS- $x G n P$ redispersed* & 91.71 & 6.44 & 0.79 & & 0.20 \\
\hline PEI-xGnP & 91.84 & 4.73 & & 3.19 & 0.25 \\
\hline PEI-xGnP redispersed* & 90.69 & 5.79 & & 3.24 & 0.28 \\
\hline
\end{tabular}

${ }^{*}$ The $\mathrm{xGnP}$ was redispersed in DI water with mild sonication for 10-15 minutes, and then filtrated and dried before XPS measurement.

TABLe 3: Zeta potential of $\mathrm{xGnP}$ before and after the noncovalent attachment of polymers.

\begin{tabular}{lc}
\hline Materials & Zeta potential $(\mathrm{mV})$ \\
\hline $\mathrm{xGnP}$ & $-32.33 \pm 1.90$ \\
SPS-xGnP & $-68.6 \pm 0.75$ \\
PEDT/SPS-xGnP & $-52.25 \pm 1.49$ \\
PDAC-xGnP & $49.39 \pm 0.45$ \\
PEI-xGnP & $48.56 \pm 1.01$ \\
\hline
\end{tabular}

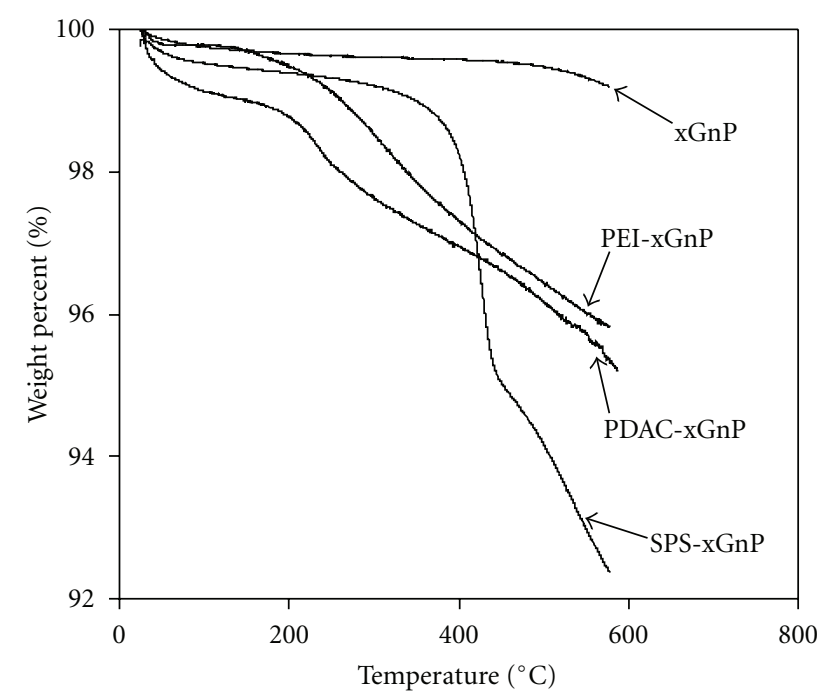

Figure 3: Thermogravimetric analysis of $\mathrm{xGnP}$ and polyelectrolytes modified $\mathrm{xGnP}$.

value of the zeta potential, the more stable the particle suspension will be. In addition, the high surface charge of the modified graphite may count the strong van der Waals force between graphite nanoplatelets to prevent agglomeration during self-assembly. As shown in Table 3, the zeta potential for the unmodified $\mathrm{xGnP}$ was measured to be $-32.33 \mathrm{mV}$, which is consistent with the literature value for the graphite particles [31]. The high absolute value of zeta potential for $\mathrm{xGnP}$ is possibly attributed to the naturally existing functional groups at the edges, such as carboxylic acid, hydroxyl, and so forth. The SPS and PEDT/SPS coated $x \mathrm{GnP}$ are kept negatively charged in aqueous solution, but the absolute value of zeta potential is increased to $68 \mathrm{mV}$ for SPS coated $\mathrm{xGnP}$, which confirms the significant adsorption of

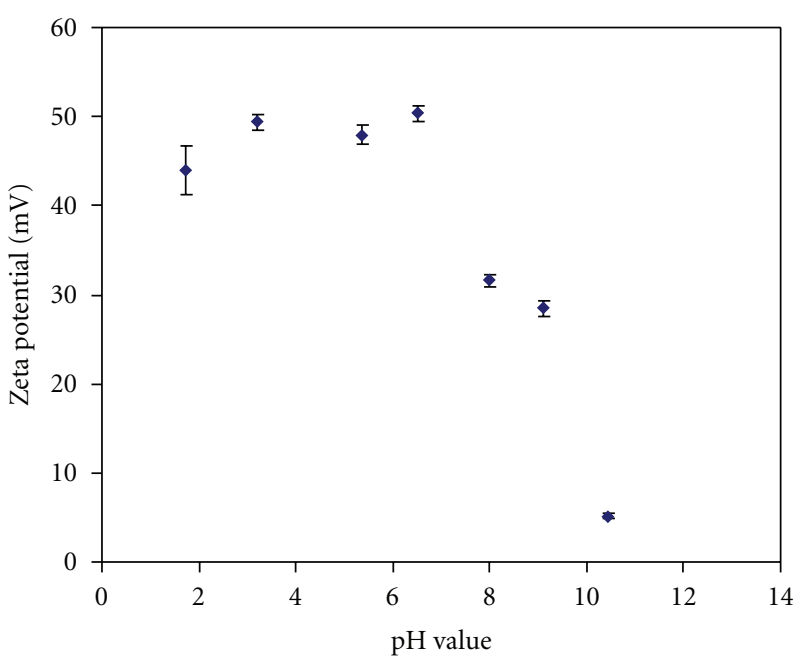

Figure 4: Zetal potential versus $\mathrm{pH}$ value for PEI coated $\mathrm{xGnP}$.

SPS at the graphite surface. The zeta potential of PEDT/SPS coated $\mathrm{xGnP}$ is slightly lower $(-52.25 \mathrm{mV})$, which is possibly due to the PEDT component in the conductive blend. For PDAC or PEI coated $\mathrm{xGnP}$, the sign of the zeta potentials changed from negative to positive, and the magnitude of the positive zeta potential increased. By a simple comparison of the absolute values of zeta potential, the SPS coated $\mathrm{xGnP}$ suspension shows better stability than $\mathrm{xGnP}$ coated with other polyelectrolytes. The zeta potentials of PEI coated nanoplatelets varied with the $\mathrm{pH}$ value of the solution, thus the suspension stability varied. As shown in Figure 4, the zeta potential of PEI coated $\mathrm{xGnP}$ keeps relatively constant at a value of $48 \mathrm{mV}$ when the $\mathrm{pH}$ is below 7, because the PEI molecules are mostly protonated which results strong repulsive force between the charged segments $\left(\mathbf{p K}_{\mathrm{a}}\right.$ of PEI is greater than 8.0). When the $\mathrm{pH}$ value is above 7 , the zeta potential significantly decreases with increasing $\mathrm{pH}$ value due to uncharged PEI molecules. At the $\mathrm{pH}$ value of 10.5, the PEI coated $\mathrm{xGnP}$ has no net charge. The stability of $\mathrm{xGnP}$ suspension is fully correlated with the zeta potential of particles. Figure 5 shows the PEI coated $\mathrm{xGnP}$ dispersed in DI water with adjustment to the varied $\mathrm{pH}$ values after sitting for 24 hours. The suspension was relatively stable when the $\mathrm{pH}$ value is close to 7 or below. However, when the $\mathrm{pH}$ value is above 10 , the graphite nanoplatelets completely settle out of solution. 


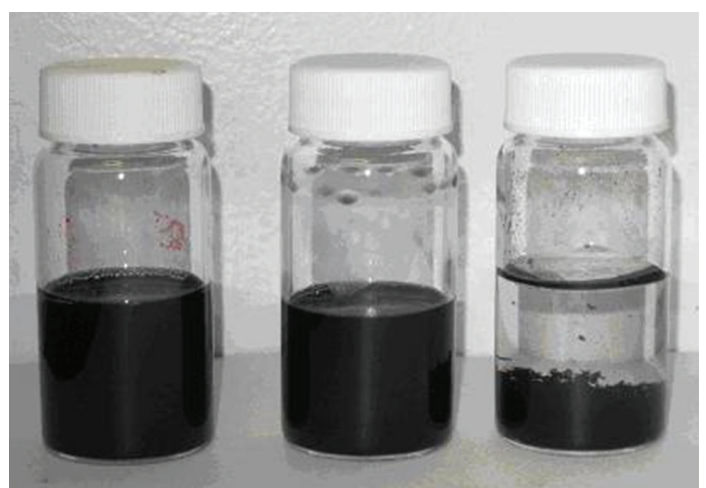

(a)

(b)

(c)

Figure 5: The stability of PEI coated $\mathrm{xGnP}$ at different solution $\mathrm{pH}$ values. (a) 5.33 , (b) 7.16 , and (c) 10.46 .

The stability of $\mathrm{xGnP}$ suspension was further quantified by measuring the concentration difference of suspension and corresponding particles size before and after sitting for 48 hours. As discussed before, when the zeta potential of particle is above $35 \mathrm{mV}$, the repulsive force between particles can overcome the attractive van der Waals force, and the agglomeration of particles can be prevented. For graphite nanoplatelets, zeta potential may not fully characterize the stability of suspension, because the high zeta potential could mostly be attributed to the edge effect, and the large hydrophobic basal plane can be attracted by each other. In addition, the surface charge is limited and may not fully account for the particle size. The comparison of the $\mathrm{xGnP}$ concentration before and after allowing the suspension to sit for 48 hours is a good indication of the stability. For both SPS and PEI coated $x G n P$, approximately $45 \%$ of $x \mathrm{GnP}$ still remain in the solution and the other settles down. The top layer suspension is very stable, no further sedimentation occurs after a week. The analysis of average particle size by dynamic light scattering gives further insight on this. The average particle size of $\mathrm{xGnP}$ was measured to be $911.3 \pm$ $47.8 \mathrm{~nm}$. Although the principles of dynamic light scattering assume the particles measured to be spherical, the measured particle size of $\mathrm{xGnP}$ is close to the one observed with microscopic technique. In addition, a different technique was used to measure the particle size using AcoustoSizer IIs (Colloidal Dynamics), which uses electroacoustic signal to determine the dynamic mobility of the particles in a colloidal suspension. It gave a similar particle size for $\mathrm{xGnP}$ (data not shown). The particle size distribution shows that $\mathrm{xGnP}$ has approximately $30 \%$ which has a particle size above $1 \mu \mathrm{m}$ (data not shown). In the top solution after sitting for 48 hours, the average particle size was measured to be $442.1 \pm$ $13.1 \mathrm{~nm}$, and $98 \%$ of particle is below $634.9 \mathrm{~nm}$, meaning that most big particles are eliminated. We have also looked at the particle size of $\mathrm{xGnP}$ for a highly stable supernatant after centrifuge, which shows a value of $200.9 \pm 6.2 \mathrm{~nm}$. Therefore, the stability of $\mathrm{xGnP}$ suspension is determined by both surface charge and particle size.

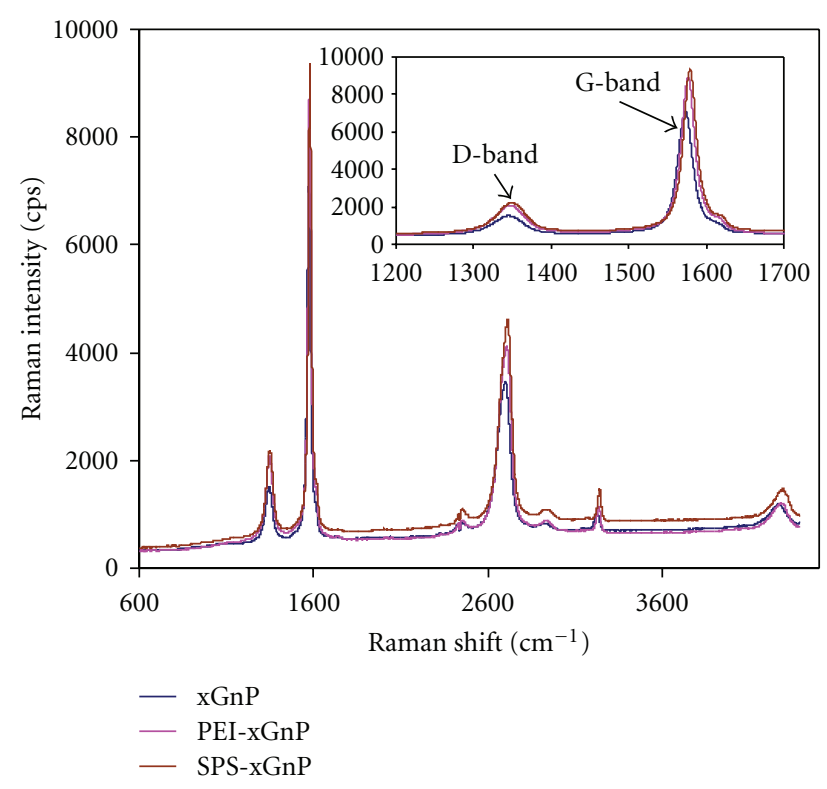

FIGURE 6: Raman spectra of $\mathrm{xGnP}$ before and after modification with polyelectrolytes (inset: enlargement of D-band and G-band).

The bonding strength of the polymer chains to the surface of $\mathrm{xGnP}$ can be measured by Raman spectrometry, because the presence of the polymer can affect the movement of carbon atoms [32]. The Raman spectra of $\mathrm{xGnP}$ and polymer coated $\mathrm{xGnP}$ are shown in Figure 6. The $\mathrm{xGnP}$ shows typical bands of graphite in the presence of disorder $[33,34]$. The band at $1575 \mathrm{~cm}^{-1}$ is so called G-band resulting from the doubly degenerate zone center $\mathrm{E}_{2 \mathrm{~g}}$ mode [33]. The peak in the $1300-1400 \mathrm{~cm}^{-1}$ region is the disorder peak known as the D-band, which is attributed to scattering from $\mathrm{sp}^{2}$ carbons containing defects. The peak between 2700 and $2800 \mathrm{~cm}^{-1}$ is $\mathrm{D}^{*}$ mode, which is an overtone of the $\mathrm{D}$ band [33]. By inspecting the spectra before and after polymer coating, it was found that both the intensity and spectral position of $\mathrm{D}$ band and $\mathrm{G}$ band have changed. In the presence of both PEI and SPS, the D band is slightly shifted to higher wave numbers, approximately $2-4 \mathrm{~cm}^{-1}$, and the position of the $\mathrm{G}$ band is shifted $3-6 \mathrm{~cm}^{-1}$ higher. The intensity and sharpness of the D band and G band increase; in addition, the ratio of the intensify of $\mathrm{D}$ band to that of the G-band slightly increases from 0.21 for $\mathrm{xGnP}$ to 0.23 for both PEI and SPS coated xGnP. Although the change in Raman spectra is not significant, it clearly indicates a typical noncovalent modification of the graphene sheet [32]. The upshift of the D and G band is possibly due to the hydrophobic and van der Waals forces between the polymer and the graphite sheet, which increase the energy necessary for vibrations to occur. And the slight increase in disorder structure after polymer coating is possibly due to the remaining carbon atoms from the polymer coating [32] and the field disturbance and physical strain in the graphene sheet caused by the interactions with the polymers. It was also noticed that the peak shift and intensity increase in Raman spectrum for SPS coated $\mathrm{xGnP}$ is more significant 


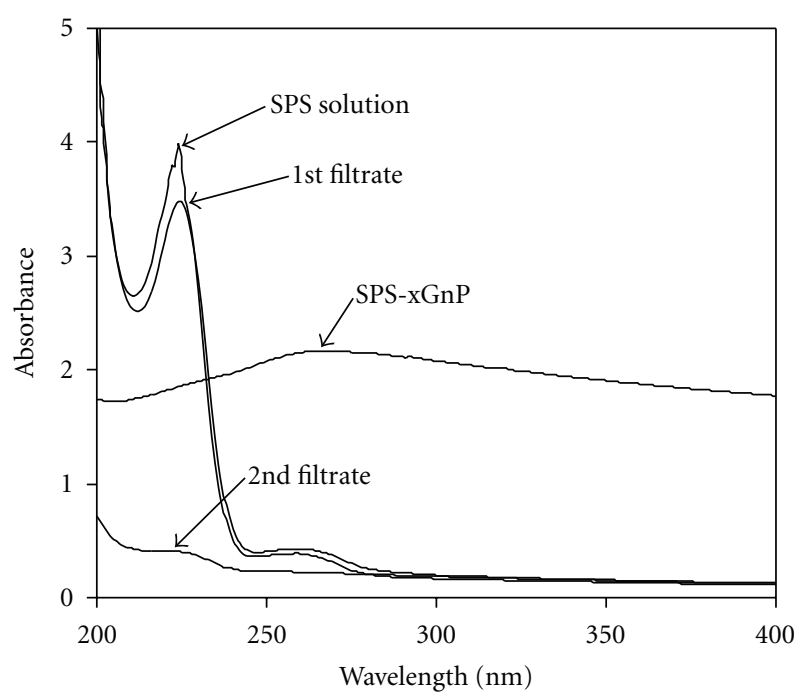

FIgUre 7: The UV-Vis spectra of SPS, SPS-xGnP and the filtrates obtained during the filtration process.

than those for PEI coated $x G n P$, which implies that SPS has a stronger interaction force with the graphene surface than PEI. This is possibly due to the molecular similarity of SPS to the graphene structure. At a high solution $\mathrm{pH}$, where PEI is unprotonated, hydrophobic or Van der Waals forces could be the main driving force. During adsorption, PEI chains can be arranged on the hydrophobic basal plane in an extended conformation [35]. Although single molecules were found on the graphite surface, it is more possible for agglomerations form due to the lack of repulsive force between the molecules [30].

Most work reported so far regarding the self-assembly of carbon nanotubes using polymer noncovalent bonding involving the simple dispersion of carbon nanotubes in polyelectrolyte solution by sonication with no further purification $[36,37]$. In this work, we found that the excess polyelectrolyte in the $\mathrm{xGnP}$ suspension plays a significant role in the self-assembly process. LbL assembly typically involves diffusion and adsorption processes, in which size matters. With the excess free polyelectrolytes, the surface coverage of $\mathrm{xGnP}$ on the glass slide was low. Therefore, it is necessary to remove the excess PEs that was not attached to xGnP. The stability of the adsorbed PE chains on graphite during filtration, the subsequent rinsing with water and redispersion utilizing sonication were examined with UV-vis spectroscopy and XPS. As shown in Figure 7, the spectrum of SPS shows a typical absorption peak at $222 \mathrm{~nm}$, and the peak height of 1st filtrate after coating with $\mathrm{xGnP}$ decreased, indicating some SPS is deposited on $\mathrm{xGnP}$. The SPS coated $\mathrm{xGnP}$ was rinsed with DI water for three times, and then redispersed in DI water by mild sonication. The second filtrate shows a very small peak at $222 \mathrm{~nm}$, which could result from the incomplete wash or dissociation of polymer due to sonication. Surprisingly, there is no SPS peak showing in the spectrum of SPS-xGnP, which is possible due to the extremely low concentration of $\mathrm{xGnP}$ in the sample. XPS was further used to confirm that SPS remains on the $\mathrm{xGnP}$ after the washing and sonication process. As shown in Table 2, the S2p atom content for SPS- $\mathrm{XGnP}$ after redispersing with sonication is close to the one before, indicating there is no loss of SPS from $\mathrm{xGnP}$ during washing and redispersion. The same result is observed for PEI-xGnP, indicated by the N1s content.

LbL self-assembly is a versatile technique to produce robust films with precise control on the film thickness and properties. With their one dimensional nanometer size, electrostatically charged PE coated surface, and their ability to form a stable colloidal dispersion, the PE modified graphite nanoplatelets are ideal candidates for multilayer self-assembly. SPS coated $\mathrm{xGnP}$ combined with PDAC was used to demonstrate the LbL assembly process. Optical microscopy images (not shown) of a single bilayer of PDAC/SPS coated $\mathrm{xGnP}$ show that the surface coverage of $\mathrm{xGnP}$ is low, possibly due to the large particle size, limiting the diffusion and repulsive electrostatic forces. With further multilayer growth, more graphite particles fill into the gaps or stack on the existing particles. At 10 bilayers, the surface became visibly black. Figures 8 and 9 show the SEM images of 4 and $10 \mathrm{xGnP}$ bilayers, respectively. At low magnification, Figure 8(a) shows most agglomerates of graphite particle, and they seem isolated. At high magnification (Figures $8(\mathrm{~b})$ and $8(\mathrm{c})$ ), it is clear that these graphite agglomerates are bridged with graphene platelets with nanometer thicknesses. The edges of agglomerates and nanoplatelets are curled or folded which disrupts the packing of $\mathrm{xGnP}$. Figure 9 shows a relatively dense packing of $\mathrm{xGnP}$ at 10 bilayers, but with the varying size and shape of the platelets, the surface of multilayer film is very rough.

The AFM image in Figure 10 shows a clear layered structure of graphene sheets, and all the nanoplatelets are stacking together and relatively close to one another. The roughness analysis of the AFM image shows a very rough surface of the multilayer film, and the section analysis shows that the thickness of 10 bilayers is in the range of 400 to $800 \mathrm{~nm}$, which is much thicker than the theoretical value of 10 bilayers. $\mathrm{xGnP} /$ polyelectrolytes multilayers can also be fabricated with PEI-xGnP and SPS as the counter ion. It was found the surface coverage of $\mathrm{xGnP}$ was much lower than SPS-xGnP/PDAC multilayers (images not shown), which is possible due to the lower surface charge of PEI-xGnP, limiting their ability to be adsorbed on the surface.

One possible application of $\mathrm{xGnP}$ thin film is as an inexpensive conductive coating. Thus, the resistance and transmittance of $\mathrm{xGnP}$ multilayers were measured. Figure 11 shows the resistance of the glass slides coated with PDAC/SPS coated $\mathrm{xGnP}$ multilayer. The $\log$ (resistance) at $1 \mathrm{~Hz}$ for the glass slide is 11.13 , which is nonconductive. Glass slides coated with PDAC/SPS coated $\mathrm{xGnP}$ are non conductive until 4 bilayers, indicating that a percolation threshold is reached. This result is consistent with the $\mathrm{OM}$ and SEM observation. The resistance of the films continually decreases with more multilayers deposited on the surface. The $\log$ (resistance) at $1 \mathrm{~Hz}$ reaches 4.85 for 10 PDAC/SPS$\mathrm{xGnP}$ bilayer film. Recently, transparent conductive carbon 


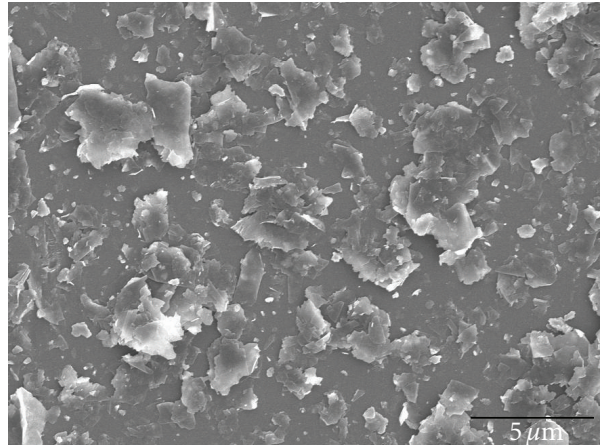

(a)

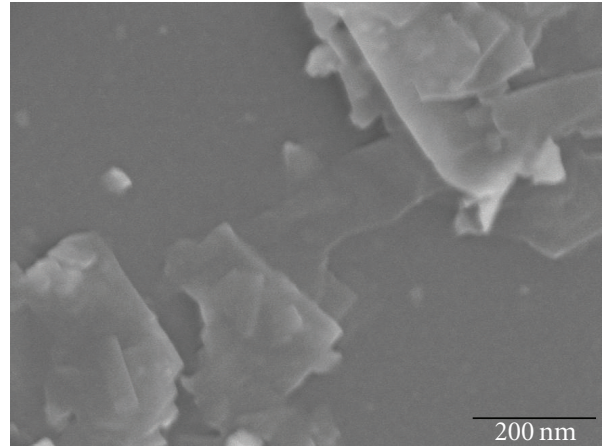

(b)

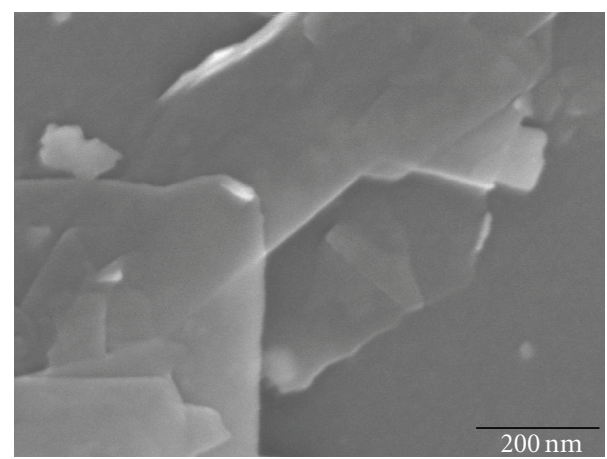

(c)

Figure 8: SEM images of 4 bilayers of PDAC/SPS-xGnP (a) low magnification (scale bar: $5 \mu \mathrm{m}$ ), (b) and (c) high magnification (scale bar: $200 \mathrm{~nm}$ ).

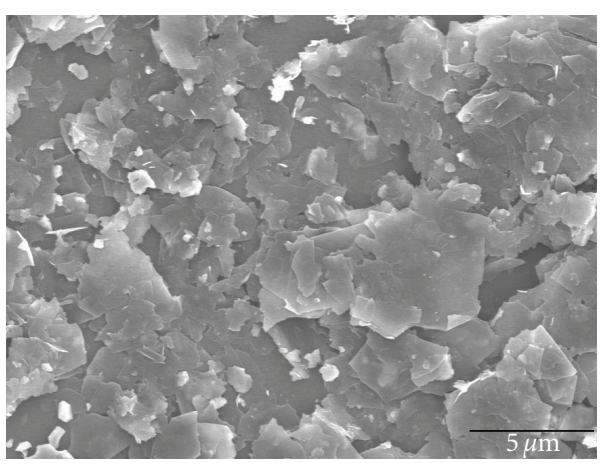

(a)

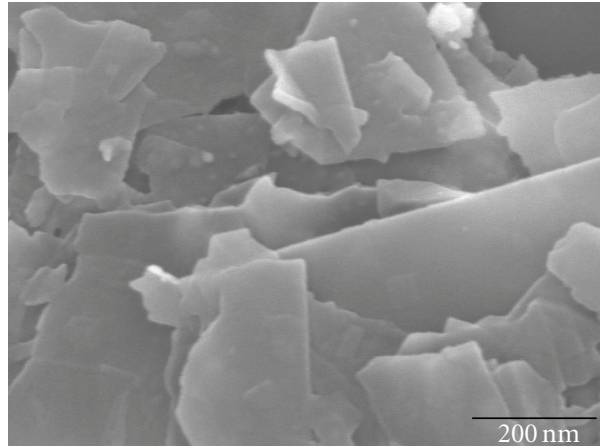

(b)

FIGURE 9: SEM images of 10 bilayers of PDAC/SPS-xGnP (a) low magnification (scale bar: $5 \mu \mathrm{m}$ ), and (b) high magnification (scale bar: $200 \mathrm{~nm})$.

nanotube films have attracted much attention due to their possible application in modern technology, such as video displays, solar cells, lasers, optical communication devices, and solid state lighting [36, 38-40]. We are pursuing the replacement of carbon nanotubes with inexpensive $\mathrm{xGnP}$ in these applications. Figure 12 shows the transmittance of $\mathrm{xGnP}$ multilayers which were measured by the UVvis spectrometer at $500 \mathrm{~nm}$ wavelength. Unfortunately, the increase in the conductivity of the film is accompanied with a dramatic decrease in the transmittance of the glass slides. The transmittance of the glass slide coated on both sides is $11.89 \%$ when the surface is conductive ( 4 bilayers), and the transmittance decreases to $2.74 \%$ at 10 bilayers. The loss of transmittance suffers from the way that graphite deposited on the surface; curled and turned-in edges of the sheets, and the agglomeration of nanoplatelets cause the high thickness of the layers. Further work is being conducted to increase the conductivity and transmittance of $\mathrm{xGnP}$ containing films using different preparation techniques, such as Langmuir-Blodgett methods and filtration methods. Patterned conductive multilayered $\mathrm{xGnP}$ composite film was also developed for applications in electronic devices [41]. 


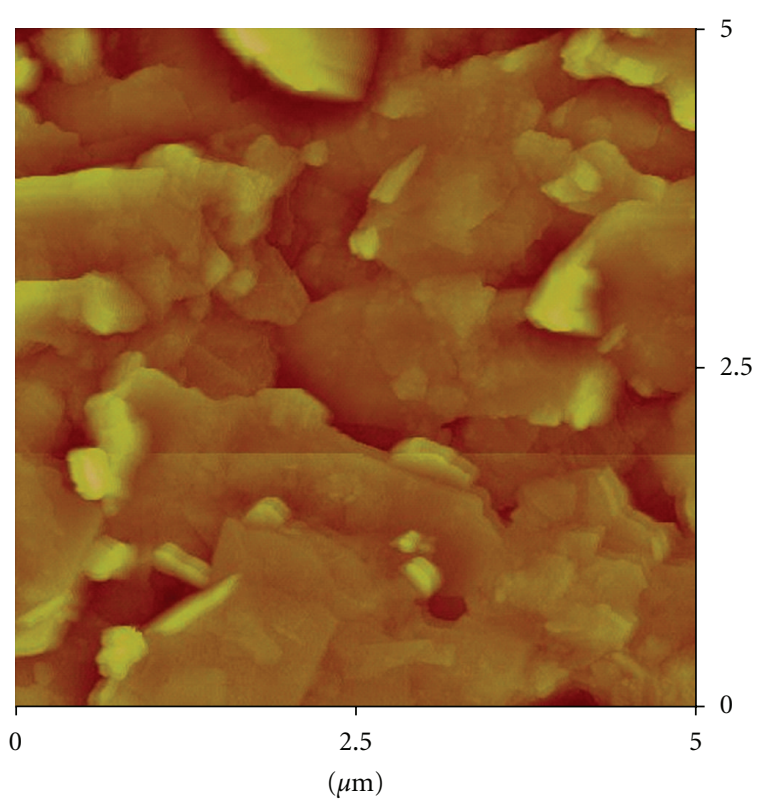

Figure 10: Height AFM image of 10 bilayers of PDAC/SPS-xGnP (color scale: black to bright yellow, $1200 \mathrm{~nm}$ ).

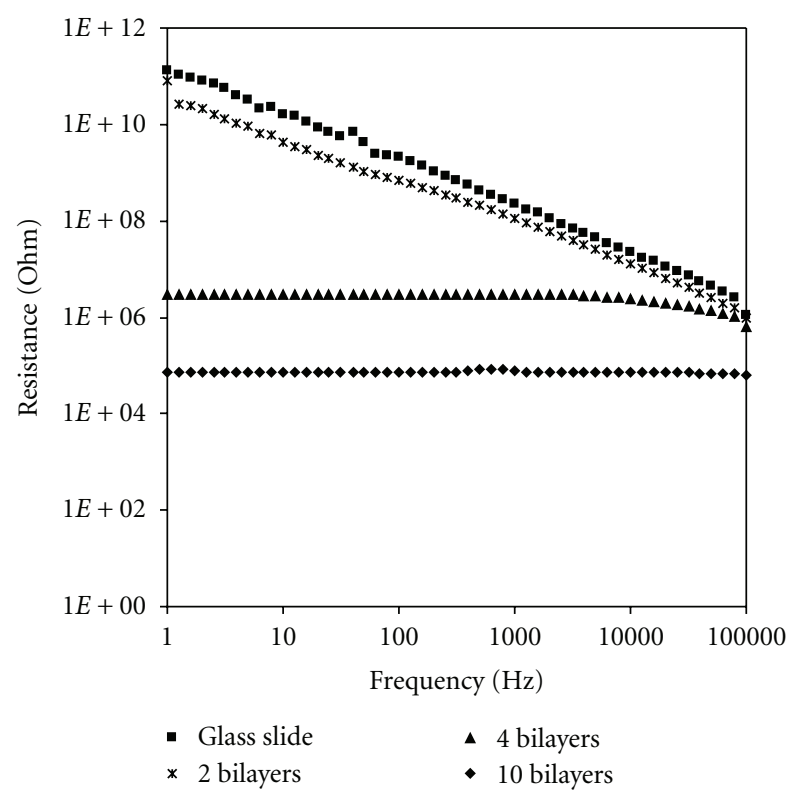

Figure 11: Resistance measurement of glass slide and xGnP multilayers.

\section{Conclusions}

In summary, we have achieved the stable aqueous suspension of graphite nanoplatelets, which are an inexpensive nanomaterial, by the noncovalent functionalization with polyelectrolytes. A variety of surfactants and polyelectrolytes have been compared for their ability to suspend graphite nanoplatelets. This noncovalent method is better for preserving the mechanical and electrical properties of graphite platelets compared to the traditional oxidation methods.

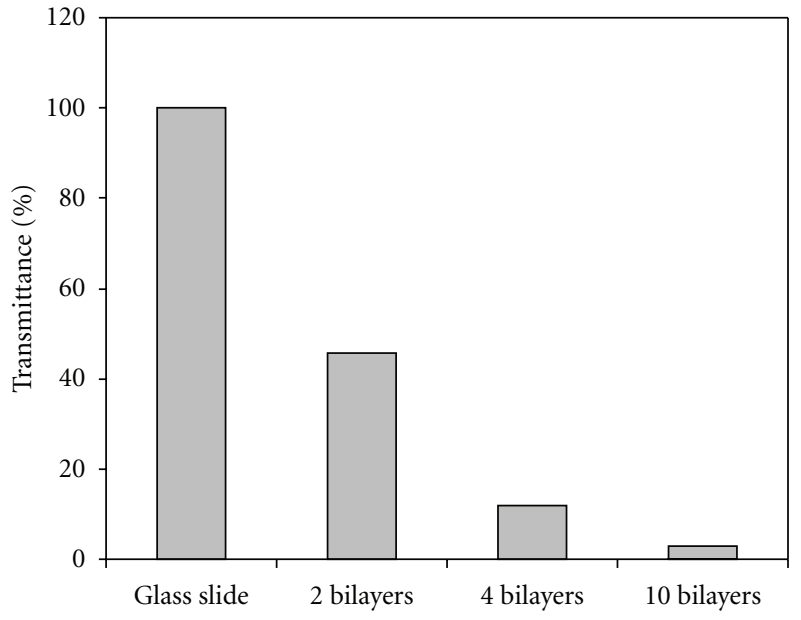

FIGURE 12: Transmittance of glass slides coated on both sides with various bilayers of PDAC/SPS-xGnP.

This robust method opens up the possibility of using these inexpensive nanomaterials in many applications, including electrochemical devices, and provides the way to processing techniques such as LbL deposition. We have demonstrated that conductive coating can be achieved by LbL assembly of polyelectrolyte-functionalized graphite nanoplatelets. Multilayer PDAC/SPS-xGnP film deposition reached the percolation threshold in only 4 cycles, resulting in a conductive thin film. The resistance of the film was lowered with more deposition cycles. However, the average roughness of these multilayer films was high, and the thickness of films was higher than theoretically estimated. This occurred because the edges of graphite nanoplatelets fold which affects the dense packing of the nanoplatelets; in addition, the nanoplatelets tend to overlap with each other. This also attributes to the low surface resistance and transmittance of $\mathrm{xGnP}$ films. Further work is on going to increase the film conductivity and transmittance using different processing methods, such as Langmuir-Blodgett and filtration.

\section{Acknowledgment}

The funding from the National Science Foundation (CBET0609164, CMMI-0832730), the University Research Corridor, the Michigan Economic Development Corporation, and the MSU Foundation to support this research is greatly appreciated.

\section{References}

[1] P. T. Hammond, "Form and function in multilayer assembly: new applications at the nanoscale," Advanced Materials, vol. 16, no. 15, pp. 1271-1293, 2004.

[2] K. Ishibashi, S. Moriyama, D. Tsuya, T. Fuse, and M. Suzuki, "Quantum-dot nanodevices with carbon nanotubes," Journal of Vacuum Science and Technology A, vol. 24, no. 4, pp. 13491355, 2006.

[3] M. Lee, J. Im, B. Y. Lee et al., "Linker-free directed assembly of high-performance integrated devices based on nanotubes and 
nanowires," Nature Nanotechnology, vol. 1, no. 1, pp. 66-71, 2006.

[4] K. Lee, J. Zhang, H. Wang, and D. P. Wilkinson, "Progress in the synthesis of carbon nanotube- and nanofiber-supported $\mathrm{Pt}$ electrocatalysts for PEM fuel cell catalysis," Journal of Applied Electrochemistry, vol. 36, no. 5, pp. 507-522, 2006.

[5] C. Lynam, S. E. Moulton, and G. G. Wallace, "Carbonnanotube biofibers," Advanced Materials, vol. 19, no. 9, pp. 1244-1248, 2007.

[6] Y. Lin, W. Yantasee, and J. Wang, "Carbon nanotubes (CNTs) for the development of electrochemical biosensors," Frontiers in Bioscience, vol. 10, pp. 492-505, 2005.

[7] C. M. Welch and R. G. Compton, "The use of nanoparticles in electroanalysis: a review," Analytical and Bioanalytical Chemistry, vol. 384, no. 3, pp. 601-619, 2006.

[8] A. C. Ferrari, J. C. Meyer, V. Scardaci et al., "Raman spectrum of graphene and graphene layers," Physical Review Letters, vol. 97, no. 18, Article ID 187401, 4 pages, 2006.

[9] A. K. Geim and K. S. Novoselov, "The rise of graphene," Nature Materials, vol. 6, no. 3, pp. 183-191, 2007.

[10] X. Li, W. Cai, J. An et al., "Large-area synthesis of high-quality and uniform graphene films on copper foils," Science, vol. 324, no. 5932, pp. 1312-1314, 2009.

[11] D. D. L. Chung, "Exfoliation of graphite," Journal of Materials Science, vol. 22, no. 12, pp. 4190-4198, 1987.

[12] W.-X. Chen, J. Y. Lee, and Z. Liu, "Preparation of Pt and PtRu nanoparticles supported on carbon nanotubes by microwaveassisted heating polyol process," Materials Letters, vol. 58, no. 25, pp. 3166-3169, 2004.

[13] H. Fukushima, "Graphite nanoreinforcements in polymer nanocomposites," in PhD Dissertation in Department of Chemical Engineering and Materials Science, Michigan State University, East Lansing, Mich, USA, 2003.

[14] S. Stankovich, D. A. Dikin, G. H. B. Dommett et al., "Graphene-based composite materials," Nature, vol. 442, no. 7100, pp. 282-286, 2006.

[15] I.-H. Do, "Metal decoration of exfoliated graphite nanoplatelets (xGnP) for fuel cell applications," in $P h D$ Dissertation in Department of Chemical Engineering and Materials Science, Michigan State University, East Lansing, Mich, USA, 2006.

[16] J. Lu, I. Do, L. T. Drzal, R. M. Worden, and I. Lee, "Nanometal-decorated exfoliated graphite nanoplatelet based glucose biosensors with high sensitivity and fast response," ACS Nano, vol. 2, no. 9, pp. 1825-1832, 2008.

[17] J. Lu, L. T. Drzal, R. M. Worden, and I. Lee, "Simple fabrication of a highly sensitive glucose biosensor using enzymes immobilized in exfoliated graphite nanoplatelets Nafion membrane," Chemistry of Materials, vol. 19, no. 25, pp. 6240-6246, 2007.

[18] W. Zhao, C. Song, and P. E. Pehrsson, "Water-soluble and optically $\mathrm{pH}$-sensitive single-walled carbon nanotubes from surface modification," Journal of the American Chemical Society, vol. 124, no. 42, pp. 12418-12419, 2002.

[19] E. T. Mickelson, I. W. Chiang, J. L. Zimmerman et al., "Solvation of fluorinated single-wall carbon nanotubes in alcohol solvents," Journal of Physical Chemistry B, vol. 103, no. 21, pp. 4318-4322, 1999.

[20] V. C. Moore, M. S. Strano, E. H. Haroz et al., "Individually suspended single-walled carbon nanotubes in various surfactants," Nano Letters, vol. 3, no. 10, pp. 1379-1382, 2003.

[21] G. Decher, "Fuzzy nanoassemblies: toward layered polymeric multicomposites," Science, vol. 277, no. 5330, pp. 1232-1237, 1997.
[22] X. Wang, H.-X. Huang, A.-R. Liu et al., "Layer-by-layer assembly of single-walled carbon nanotube-poly(viologen) derivative multilayers and their electrochemical properties," Carbon, vol. 44, no. 11, pp. 2115-2121, 2006.

[23] T. Cassagneau and J. H. Fendler, "High density rechargeable lithium-ion batteries self-assembled from graphite oxide nanoplatelets and polyelectrolytes," Advanced Materials, vol. 10, no. 11, pp. 877-881, 1998.

[24] T. Cassagneau, F. Guérin, and J. H. Fendler, "Preparation and characterization of ultrathin films layer-by-layer selfassembled from graphite oxide nanoplatelets and polymers," Langmuir, vol. 16, no. 18, pp. 7318-7324, 2000.

[25] N. A. Kotov, I. Dékány, and J. H. Fendler, "Ultrathin graphite oxide-polyelectrolyte composites prepared by self-assembly: transition between conductive and non-conductive states," Advanced Materials, vol. 8, no. 8, pp. 637-641, 1996.

[26] N. I. Kovtyukhova, P. J. Ollivier, B. R. Martin, et al., "Layer-bylayer assembly of ultrathin composite films from micron-sized graphite oxide sheets and polycations," Chemistry of Materials, vol. 11, no. 3, pp. 771-778, 1999.

[27] L. T. Drzal and H. Fukushima, "Expanded graphite and products produced therefrom," 2004, US Patent application 20040127621.

[28] W. N. Reynolds, Physical Properties of Graphite, Elsevier, Amsterdam, The Netherlands, 1968.

[29] S. Stankovich, R. D. Piner, X. Chen, N. Wu, S. T. Nguyen, and R. S. Ruoff, "Stable aqueous dispersions of graphitic nanoplatelets via the reduction of exfoliated graphite oxide in the presence of poly(sodium 4-styrenesulfonate)," Journal of Materials Chemistry, vol. 16, no. 2, pp. 155-158, 2006.

[30] M. Schneider, M. Brinkmann, and H. Möhwald, "Adsorption of polyethylenimine on graphite: an atomic force microscopy study," Macromolecules, vol. 36, no. 25, pp. 9510-9518, 2003.

[31] V. K. Paruchuri, A. V. Nguyen, and J. D. Miller, "Zetapotentials of self-assembled surface micelles of ionic surfactants adsorbed at hydrophobic graphite surfaces," Colloids and Surfaces A, vol. 250, no. 1-3, pp. 519-526, 2004.

[32] V. A. Sinani, M. K. Gheith, A. A. Yaroslavov et al., "Aqueous dispersions of single-wall and multiwall carbon nanotubes with designed amphiphilic polycations," Journal of the American Chemical Society, vol. 127, no. 10, pp. 3463-3472, 2005.

[33] S. Reich and C. Thomsen, "Raman spectroscopy of graphite," Philosophical Transactions of the Royal Society A, vol. 362, no. 1824, pp. 2271-2288, 2004.

[34] F. Tuinstra and J. L. Koenig, "Raman spectrum of graphite," Journal of Chemical Physics, vol. 53, no. 3, pp. 1126-1130, 1970.

[35] J. Park and P. T. Hammond, "Polyelectrolyte multilayer formation on neutral hydrophobic surfaces," Macromolecules, vol. 38, no. 25, pp. 10542-10550, 2005.

[36] M. Palumbo, K. U. Lee, B. T. Ahn et al., "Electrical investigations of layer-by-layer films of carbon nanotubes," Journal of Physics D, vol. 39, no. 14, pp. 3077-3085, 2006.

[37] H. Paloniemi, M. Lukkarinen, T. Ääritalo et al., "Layer-bylayer electrostatic self-assembly of single-wall carbon nanotube polyelectrolytes," Langmuir, vol. 22, no. 1, pp. 74-83, 2006.

[38] T. M. Barnes, X. Wu, J. Zhou et al., "Single-wall carbon nanotube networks as a transparent back contact in CdTe solar cells," Applied Physics Letters, vol. 90, no. 24, Article ID 243503, 3 pages, 2007. 
[39] A. Schindler, J. Brill, N. Fruehauf, J. P. Novak, and Z. Yan IV, "Solution-deposited carbon nanotube layers for flexible display applications," Physica E: Low-Dimensional Systems and Nanostructures, vol. 37, no. 1-2, pp. 119-123, 2007.

[40] Z. Wu, Z. Chen, X. Du et al., "Transparent, conductive carbon nanotube films," Science, vol. 305, no. 5688, pp. 1273-1276, 2004.

[41] T. R. Hendricks, J. Lu, L. T. Drzal, and I. Lee, "Intact pattern transfer of conductive exfoliated graphite nanoplatelet composite films to polyelectrolyte multilayer platforms," Advanced Materials, vol. 20, no. 10, pp. 2008-2012, 2008. 

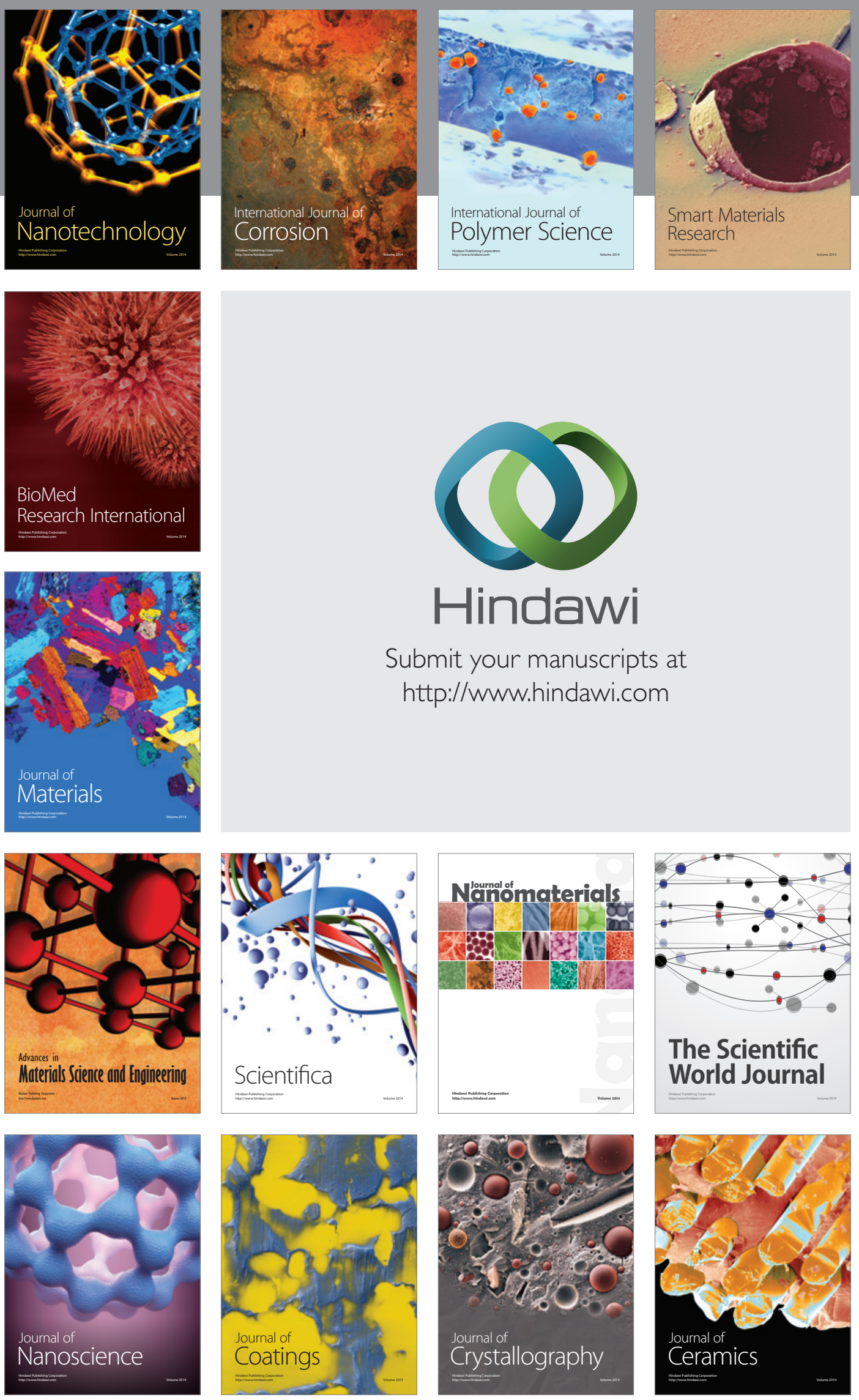

The Scientific World Journal

Submit your manuscripts at

http://www.hindawi.com

\section{World Journal}

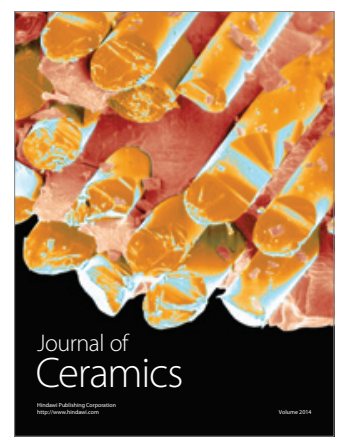

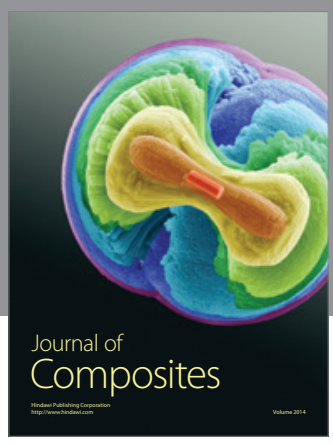
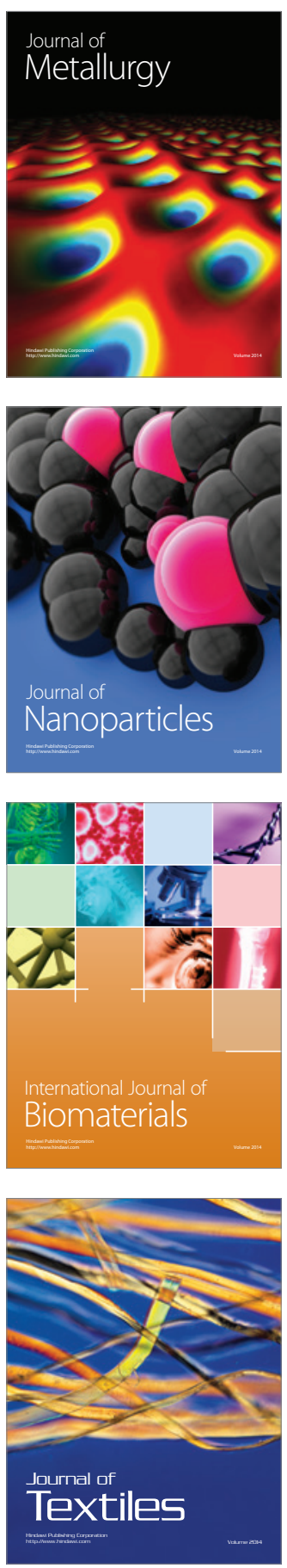